\title{
A Practical Guide to Digital Holography and Generalized Sampling
}

\author{
D. P. Kelly ${ }^{a}$, B. M. Hennelly ${ }^{a}$, C. McElhinney ${ }^{a}$, and T. J. Naughton ${ }^{a, b}$ \\ ${ }^{a}$ Department of Computer Science, National University of Ireland, \\ Maynooth, Ireland; \\ ${ }^{b}$ University of Oulu, RFMedia Laboratory, Oulu Southern Institute, \\ Vierimaantie 5, 84100 Ylivieska, Finland
}

\begin{abstract}
The theorems of Nyquist, Shannon and Whittaker have long held true for sampling optical signals. They showed that a signal (with finite bandwidth) should be sampled at a rate at least as fast as twice the maximum spatial frequency of the signal. They proceeded to show how the continuous signal could be reconstructed perfectly from its well sampled counterpart by convolving a Sinc function with the sampled signal. Recent years have seen the emergence of a new generalized sampling theorem of which Nyquist Shannon is a special case. This new theorem suggests that it is possible to sample and reconstruct certain signals at rates much slower than those predicted by Nyquist-Shannon. One application in which this new theorem is of considerable interest is Fresnel Holography. A number of papers have recently suggested that the sampling rate for the digital recording of Fresnel holograms can be relaxed considerably. This may allow the positioning of the object closer to the camera allowing for a greater numerical aperture and thus an improved range of 3D perspective. In this paper we: (i) Review generalized sampling for Fresnel propagated signals, (ii) Investigate the effect of the twin image, always present in recording, on the generalized sampling theorem and (iii) Discuss the effect of finite pixel size for the first time.
\end{abstract}

Keywords: Digital holography, phase shifting interferometry

\section{INTRODUCTION}

Holography the science of recording and reconstructing a complex electromagnetic wavefield, was invented by Gabor in 1948. ${ }^{1}$ This initial invention concerned itself with electron microscopy and predated the invention of the laser. With the onset of the laser E. Leith and J. Upatnieks ${ }^{2,3}$ appended the holographic principle with the introduction of the offset reference wave. This enabled the separation of the object wavefield from the other components that are generated in the optical reconstruction process, namely the intensities of the object and reference wavefields and also the so called 'ghost' or conjugate image. Holography may also be employed to describe the science of optical interferometry, ${ }^{4}$ which incorporates important industrial measurement techniques. We note that holography is at the heart of countless optical and non optical techniques. ${ }^{5}$

Using photosensitive recording materials to record holograms is costly and inflexible. Digital holography ${ }^{6-9}$ (DH) refers to the science of using discrete electronic devices, such as CCDs to record the hologram. In this case reconstruction is performed numerically by simulating the propagation of the wavefield back to the plane of the object. ${ }^{10-12}$ This often requires some kind of redundancy reduced fast algorithms to numerically calculate the Fresnel Transform ${ }^{13}$ in $O(N \log N)$ calculations. These algorithms are often based around the use of the Fast Fourier Transform ${ }^{14}$ algorithm developed for $O(N \log N)$ calculation of the Fourier Transform. ${ }^{13,15}$ In recent years DH has been demonstrated to be a useful method in many areas of optics such as microscopy, ${ }^{16,17}$ deformation analysis, ${ }^{18}$ object contouring, ${ }^{19}$ particles sizing and position measurement. ${ }^{20}$ 'In-line' or 'on-axis' DH refers to the implementation of the original Gabor architecture in which the reference wavefield travels in the same direction as the object wavefield. As in the continuous case this method suffers from poor reconstructed image

Further author information:

D.P.K.: E-mail: damienk@cs.nuim.ie, Telephone: 00353 (0)1 7086089

Optics and Photonics for Information Processing II, edited by Abdul Ahad Sami Awwal,

Khan M. Iftekharuddin, Bahram Javidi, Proc. of SPIE Vol. 7072, 707215, (2008)

0277-786X/08/\$18 - doi: $10.1117 / 12.795904$

Proc. of SPIE Vol. 7072 707215-1 


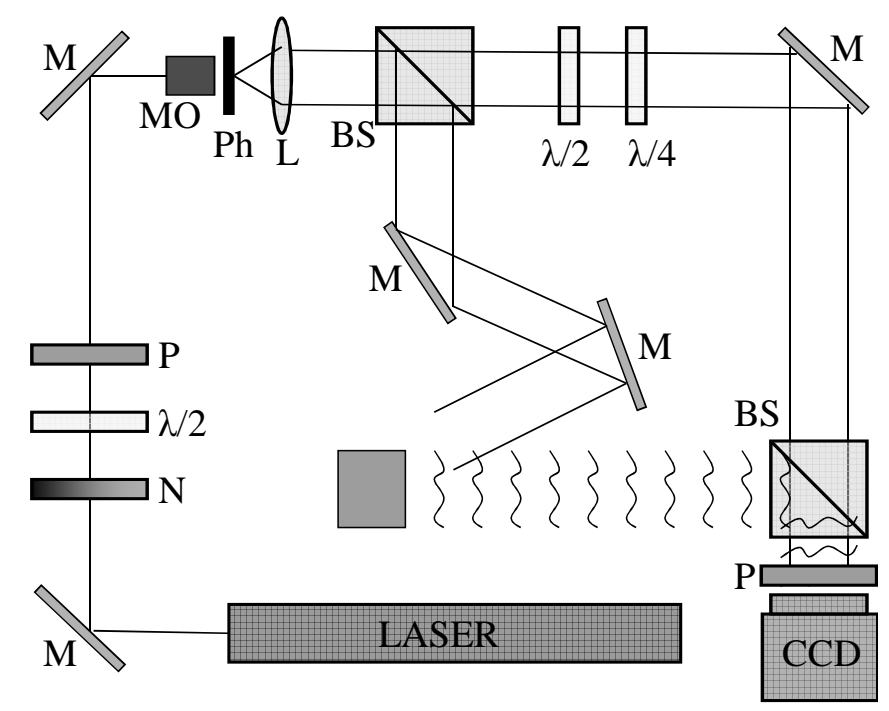

Figure 1. Digital Holography In-line Architecture. The presence of the phase retarders allow us to make multiple captures with different constant phase shifts of the reference beam. We can therefor implement the Phase Shifting Interferometry algorithm to extract the complex wavefront: MO Microscope Objective, Ph Pinhole, N Neutral Density Filter, P Polarizer, M Mirror, L Lens, BS Beam splitter, $\lambda / 2$ Have Wave Plate, $\lambda / 4$ Quarter Wave Plate

quality, due to the presence of the intensity terms and the conjugate image that contaminates the reconstructed object image. While it is possible to remove the intensity terms with efficient numerical techniques, ${ }^{21}$ it remains difficult to remove the conjugate image. This may be achieved using an off-axis recording setup equivalent to that used by Leith and Upatnieks. ${ }^{2,3}$ However, this increases the spatial resolution requirements, and limits the system significantly which is undesirable when one considers the already limited resolution of pixilated cameras. An alternative approach known as phase-shifting interferometry ${ }^{22,23}$ (PSI) has been introduced allowing for an in-line set-up to be used with at least two successive captures and enabling separation of the object wavefield from all of the other terms. In Fig. 1 we illustrate the in-line and PSI architectures.

In general all electromagnetic signals when sampled are subject to the laws devised in the first half of the last century by Nyquist, ${ }^{24}$ Shannon, ${ }^{25}$ Whittaker ${ }^{26}$ and Kotelnikov. ${ }^{27}$ Until recently it was assumed that digital holography was subject to these same laws. Simply stated the rules of sampling and interpolation are as follows: It is necessary for the signal of interest to be bounded in frequency - in our case spatial frequency. If the continuous signal is sampled at discrete intervals with a sampling rate at least as fast as twice the maximum frequency (the Nyquist rate) then the continuous signal can be reconstructed. By reconstructed we mean that the continuous signal may be 'interpolated' from the discrete sampled values by applying Shannon interpolation. This can be explained most elegantly using the Fourier Transform ${ }^{13,15}$ sampling creates an infinite number of copies of the signals fourier transform, all beside each other with a separation equal to the inverse of the sampling interval. If the sampling rate is large enough and therefore the sampling interval small enough these copies will not overlap with one another due to their finite support. The continuous signal can be simply reconstructed by isolating one of these copies, achieved by multiplying by an appropriate Rect function. Multiplying by a Rect function in the frequency domain amounts to convolving the sampled signal with a Sinc function. This convolution is often called Shannon interpolation.

Recently there has been considerable interest in the literature ${ }^{12,28-34}$ in sampling certain optical signals at a rate below the Nyquist rate and still managing to reconstruct the continuous signal perfectly. This collective work has demonstrated the property of finite support in the Fourier domain is too strict a requisite for interpolation to be achievable. It is sufficient that the signal is bounded in any one of an infinite set of domains, ${ }^{35}$ output domains of the Linear Canonical Transform (LCT). The Fourier Transform and the Fresnel Transform ${ }^{13}$ are special cases of the LCT. If the signal is bounded within some finite support in such a domain then the signal 
can be sampled at a rate proportional to LCT support width. The Nyquist sampling rate which is proportional to the Fourier support width (bandwidth) is a special case of this more generalized sampling theorem. It is possible also to deduce a more general interpolation formula. This amounts to multiplying the signal samples by an appropriate chirp function (the scale of the chirp is dependent on the LCT domain in which the signal has finite support), followed by standard Shannon interpolation and this is in turn followed by multiplying by the conjugate of the fore-mentioned chirp. If a signal has finite support in some LCT domain the, generalized sampling theorem predicts a much more relaxed sampling rate that the Nyquist Shannon theorem. This paper concerns itself with the generalized sampling theorem for the special case of a signal having finite support in the Fresnel domain.

The generalized sampling theorem for the special case of the Fresnel Transform has been proposed to have important application to Fresnel Digital Holography. ${ }^{30,31,36}$ It is of considerable interest to this research area because it seems to suggest that one may place the object to be recorded at a distance mich closer that previously defined by the Nyquist Shannon theorem. This should allow for a greater numerical aperture, which in turn should allow reconstruction of the object at a resolution previously though impossible and greater than the resolution of the recording CCD. Furthermore this should allow for a far greater range of 3D perspectives to be reconstructed. The purpose of this paper is to review the concept of generalized sampling for Fresnel Holography, to reinterpret it and to discuss and investigate some problems that we have identified as existing for a physical practical implementation of the theorem.

The outline of this paper is as follows: In Section 2 we review the generalized sampling theorem for light propagation in the paraxial regime (Fresnel Transform). In Section 2 we also discuss in detail what generalized sampling means in Fourier domain of the propagating signal. Aliasing does occur, but it can be described as a reversible encoding of the signal. In Section 3 we discuss some physical non-ideality of generalized sampling when applied to digital holography. In particular we focus two main topics - the inherent presence of the twin image in any interferogram and secondly we discuss for the first time the implications of finite pixel sizes on the theorem. The theorem assumes that sampling is performed by infinitely narrow Dirac delta functionals.

\section{GENERALIZED SAMPLING}

The purpose of this section is to theoretically examine some of the fundamental resolution limitations that occur in practical digital holographic systems. A typical DH set-up is depicted in Fig. 1 and in this section it is assumed that the complex field can be captured at the camera face using interferometic techniques. The field at the object plane is then calculated digitally using numerical techniques by a computer. Let us assume that the object field in Fig. 1 has a maximum spatial frequency given by $f_{\max }$. This field then propagates to the camera face where its complex amplitude is recorded. For now, we assume that the field at the camera plane also contains this maximum spatial frequency - later we will explicitly show that this is the case. A camera's sampling rate, $f_{c}$, and thus its ability to resolve a spatial frequency at the camera plane is determined by the distance between adjacent pixels on the camera face. Thus on initial consideration one might conclude that the camera must be able to resolve $f_{\max }$ (i.e. $f_{c} \geq 2 f_{\max }$ ) if the object field is to be reconstructed properly. However as we have noted in the introduction several authors have shown that this sampling criterion may be too strict and that it is possible to recover the object signal when it's Fresnel transform is sampled in the camera plane at a rate lower than the Nyquist limit. These results may be significant in the field of digital holography and so we set ourselves the task of investigating this further. In Section 2.1 we re-derive the theoretical result presented elsewhere ${ }^{28,29,31}$ and in Section 2.2 we explore some of the implications of these sampling rules by using a specific analytical example.

\subsection{Sampling the diffracted field}

Sampling an analytical function $f(x)$ is equivalent to multiplying that function by an infinite train of Dirac delta functions, $\delta_{T}(x),{ }^{31}$ mathematically we write

$$
\begin{aligned}
f^{s}(n) & =f(x) \delta_{T}(x) \\
f^{s}(n) & =f(n T),
\end{aligned}
$$


where $\delta_{T}(x)=\sum_{n=-\infty}^{\infty} \delta(x-n T)$ and where $T$ is the distance between samples. The sampling frequency $f_{s}$ is related to $T$ by the formula $f_{s}=1 / T$. Since the function $\delta_{T}(x)$ is periodic it may also be expressed mathematically using a Fourier series representation, ${ }^{32}$

$$
\delta_{T}(x)=\sum_{n=-\infty}^{\infty} \exp \left(j 2 \pi n f_{s} x\right) .
$$

We now wish to examine the effect of sampling a field in the diffraction plane. Let there be two fields, $u(X)$ and $u_{z}(x)$ that are related to each other by a Fresnel transform,

$$
\begin{aligned}
& u_{z}(x)=\chi_{z}\{u(X)\}(x) \\
& u_{z}(x)=\frac{1}{\sqrt{j \lambda z}} \int u(X) \exp \left[\frac{j \pi}{\lambda z}(x-X)^{2}\right] d X \\
& u(X)=\frac{1}{\sqrt{-j \lambda z}} \int u_{z}(x) \exp \left[\frac{-j \pi}{\lambda z}(X-x)^{2}\right] d x,
\end{aligned}
$$

where $\chi$ is the Fresnel transform operator. We also note the shifting property of the Fresnel transform ${ }^{28}$ for some analytical signal $f(X)$,

$$
\chi_{z}\{f(X) \exp (j 2 \pi \xi X)\}(x)=\exp \left(\frac{-j \pi \xi^{2}}{\lambda z}\right) \exp (j 2 \pi x \xi) \chi_{z}\{f(X)\}(x-\xi \lambda z) .
$$

We are interested in sampling the field in the diffraction (camera) plane and so now using Eq. (1) and Eq. (2) we rewrite Eq. (3) to give

$$
u^{s}(X)=\frac{1}{\sqrt{-j \lambda z}} \int u_{z}(x) \sum_{n=-\infty}^{\infty} \exp \left(j 2 \pi n f_{s} x\right) \exp \left[\frac{-j \pi}{\lambda z}(X-x)^{2}\right] d x
$$

We have included the superscript "s" in Eq. (5) to emphasis that it is the result of an inverse Fresnel transform of $u_{z}(x) \delta_{T}(x)$. Taking the sum outside the integral and making use of the Fresnel shift theorem, Eq. (4), we find that $u^{s}(X)$ is given by

$$
\begin{aligned}
& u^{s}(X)=\frac{1}{\sqrt{-j \lambda z}} \sum_{n=-\infty}^{\infty} \int u_{z}(x) \exp \left(j 2 \pi n f_{s} x\right) \exp \left[\frac{-j \pi}{\lambda z}(X-x)^{2}\right] \\
& u^{s}(X)=\sum_{n=-\infty}^{\infty} \chi_{-z}\left\{u_{z}(x) \exp \left[j 2 \pi\left(n f_{s}\right) x\right]\right\}(X) \\
& u^{s}(X)=\sum_{n=-\infty}^{\infty} \exp \left[\frac{-j \pi\left(n f_{s}\right)^{2}}{\lambda z}\right] \exp \left(j 2 \pi X n f_{s}\right) \chi_{-z}\left\{u_{z}(x)\right\}\left(X-n f_{s} \lambda z\right) .
\end{aligned}
$$

We note several points about this result: (i) the sampling process creates an infinite number of replicas in the object plane, (ii) each of these replicas are separated from each other by a distance $n f_{s} \lambda z$, (iii) each of the replicas is also multiplied by a linear phase as well as some unimportant constant phase.

If we impose the constraint that our object field has a finite support $\Delta$ in the object plane then this field can be perfectly reconstructed provided that $f_{s} \geq \frac{\Delta}{\lambda z}$.

\subsection{Examination of a Special Case}

Let us consider a field, $u(X)$, that has finite support, $\Delta$, at the plane: $z=0$. From the results derived in Section 2.2, we know that this field can be recovered provided that it is sampled at a rate $f_{s} \geq \frac{\Delta}{\lambda z_{c}}$ in the diffraction plane $\left(z=z_{c}\right)$ or equivalently such that $T \leq \frac{\lambda z_{c}}{\Delta}$. We make some idealizations about the nature of our camera namely: (i) the physical extent of the camera is much larger than the object extent, (ii) that the 


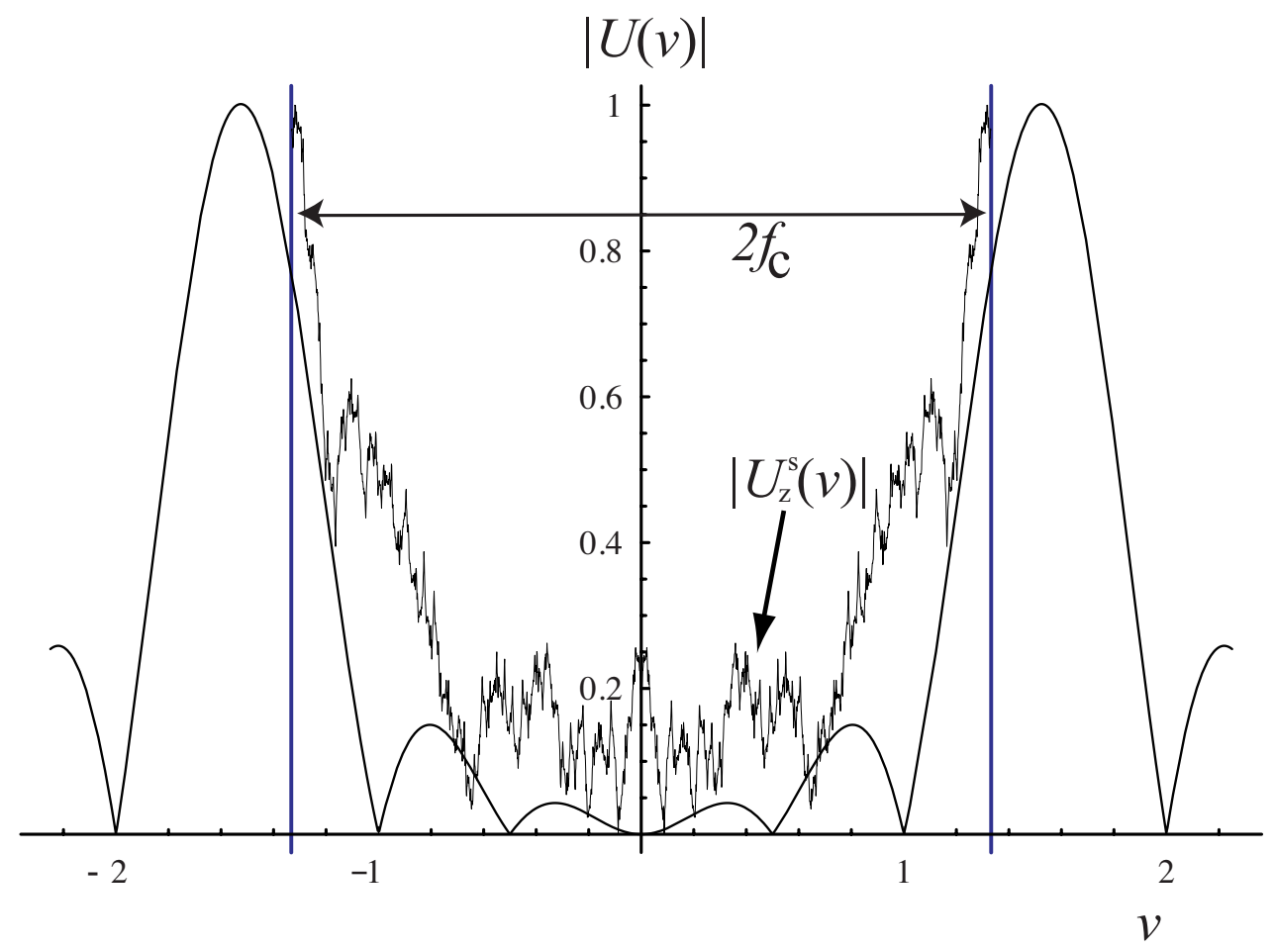

Figure 2. $|U(v)|$ as a function of $v L$ over the range $-2.2 \leq v L \leq 2.2$. The values $U_{z}^{s}(v)$ are obtained by performing a numerical Fourier transform on the field $u_{z}(x)$ when sampled at a rate $f_{c}$.

pixels act as point detectors, i.e. no intensity averaging occurs over the pixel area. For the practical digital holography system that we wish to consider it will be the camera that ultimately samples this diffracted field. If the spacing between adjacent pixels is fixed at $T$, the sampling rate at the diffraction (camera) plane is automatically defined as $2 f_{c}=\frac{1}{T}$. However before we consider how the camera samples the diffracted field let us first examine the properties of a particular field using analytical expressions to describe its spatial and spatial frequency distributions. We now define the field $u(X)$ as

$$
u(X)=\operatorname{rect}_{L}(X) \sin \left(2 \pi f_{x} X\right)
$$

and its Fourier domain representation

$$
U(v)=\left(\frac{0.5-j 0.5}{\pi^{\frac{3}{2}}}\right)\left\{\frac{\sin \left[2 \pi L\left(f_{x}-v\right)\right]}{f_{x}-v}+\frac{\sin \left[2 \pi L\left(f_{x}+v\right)\right]}{f_{x}+v}\right\},
$$

and where

$$
\operatorname{rect}_{L}(x)=\{1,|x| \leq L \text {, and } 0 \text { otherwise. }
$$

The finite support of $u(X)$ is imposed by Eq.(9). Setting $f_{x} \approx 596.2 \mathrm{~m}^{-1}, L \approx 2.52 \mathrm{~mm}$ and dropping the first constant term in the round brackets from Eq. (8), we plot the normalized $|U(v)|$ over the range $-2.2 \leq v L \leq 2.2$. We note two things in particular about the distribution in this plot: $(1)$ Since $u(X)$ has finite support, its Fourier representation $U(v)$ is not bounded, (2) there is a significant peak in the distribution at $v L=+/-1.5$ which is due to the sinusoidal nature of $u(X), f_{x} L=1.5$.

We now wish to examine how the magnitude of the spatial frequency distribution changes as the field propagates from the plane $z=0$. Following Goodman ${ }^{13}$ we can write the spatial frequency distribution of the Fresnel transformed signal as

$$
U_{z}(v)=U(v) \exp \left(-j \pi \lambda z v^{2}\right)
$$



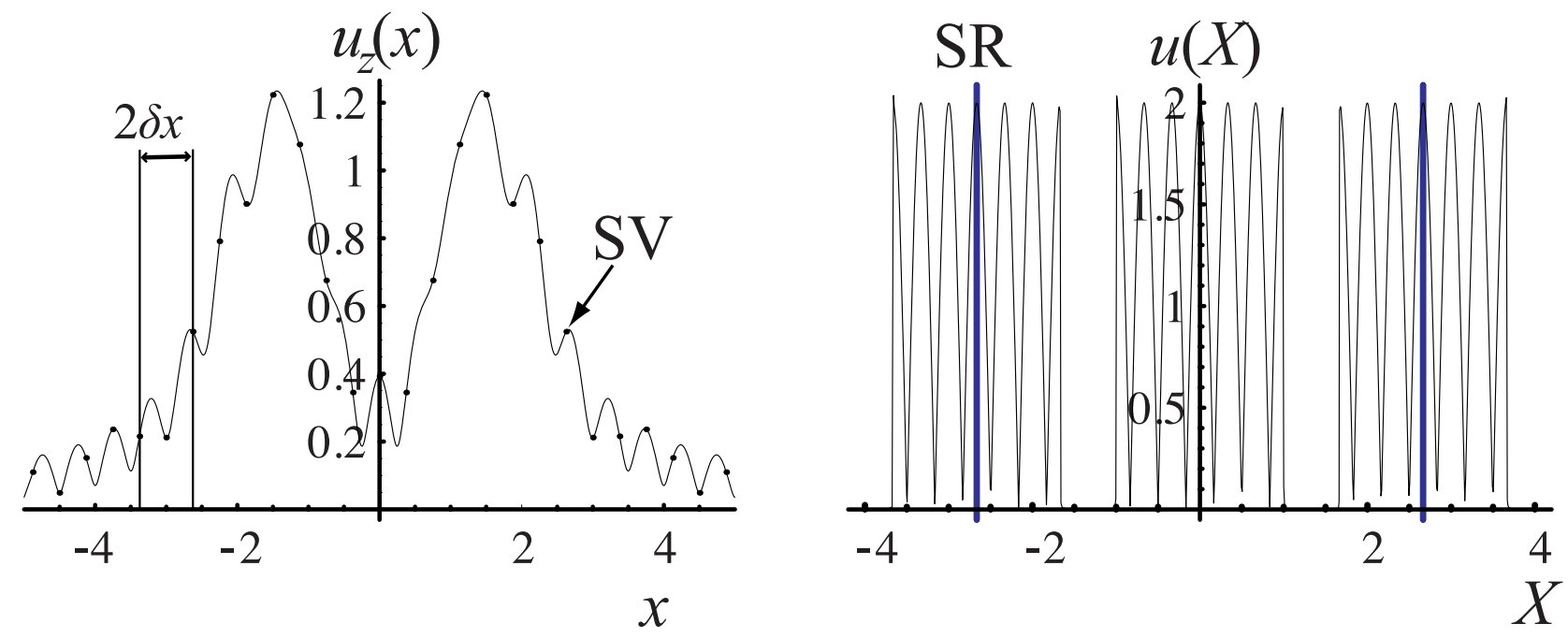

Figure 3. (a) Plot of $\left|u_{z}(x)\right|$ over the range $-5 \leq x / L \leq 5$. SV - sampled values are the complex values of $u_{z}(x)$ taken at the points indicated with the black dots. (b) Plot of $\left|u^{s}(X)\right|$ over the range $-5 \leq x / L \leq 5$. SR - sampled replica.

We note that $\left|U_{z}(v)\right|$ may be written as

$$
\begin{aligned}
\left|U_{z}(v)\right| & =\sqrt{U_{z}(v) U_{z}^{*}(v)} \\
\left|U_{z}(v)\right| & =|U(v)|
\end{aligned}
$$

where ${ }^{*}$ denotes the complex conjugate operation. It is important to note from Eq. (11) that the magnitude of the spatial frequency distribution remains invariant under propagation. This means that the field incident on the camera also contains the spatial frequencies $f_{x}$. Since the results of Section 2.1 imply that we may sample the field at a rate lower than $f_{x}$ and still recover that frequency in the object field we will now sample the this diffracted field, $u_{z}(x)$ at a sampling rate $f_{c}$ such that $f_{c} \leq f_{x}$, see Fig. 2 and show that we can recover the field in the object plane accurately. The field $u_{z}(x)$ has an analytical form given by

$$
\begin{aligned}
u_{z}(x)= & \frac{(-1)^{0.25}}{2} \exp \left[-j \pi f_{x}\left(2 x+f_{x} z \lambda\right)\right] \\
& \times\left\{\exp \left(j 4 \pi f_{x} x\right)\left[\operatorname{erfi}\left(\Theta_{1}\right)+\operatorname{erfi}\left(\Theta_{2}\right)\right]+\left[\operatorname{erfi}\left(\Theta_{3}\right)+\operatorname{erfi}\left(\Theta_{4}\right)\right]\right\},
\end{aligned}
$$

where

$$
\begin{aligned}
& \Theta_{1}=\frac{(-1)^{0.25} \sqrt{\pi}}{\sqrt{\lambda z}}\left(L+x-f_{x} z \lambda\right), \\
& \Theta_{2}=\frac{(-1)^{0.25} \sqrt{\pi}}{\sqrt{\lambda z}}\left(L-x+f_{x} z \lambda\right), \\
& \Theta_{3}=\frac{-(-1)^{0.25} \sqrt{\pi}}{\sqrt{\lambda z}}\left(-L+x+f_{x} z \lambda\right), \\
& \Theta_{4}=\frac{(-1)^{0.25} \sqrt{\pi}}{\sqrt{\lambda z}}\left(L+x+f_{x} z \lambda\right) .
\end{aligned}
$$

Setting $\lambda=633 \times 10^{-9} \mathrm{~m}$, and $z_{c}=10$, in Fig. 2 (a) we plot $\left|u_{z}(x)\right|$ over the range $-5 \leq \frac{x}{L} \leq 5$. This is the analytical function that we wish to sample. Thus we now take a series of samples (SV) of $u_{z}(x)$ [see black dots in Fig. 3(a)] spaced at regular intervals, $T / L=0.3745$, from each other as indicated in Fig. 3(a), over the range $-20 \leq \frac{x}{L} \leq 20$, [Note: In Fig. 3, we have only presented $\left|u_{z}(x)\right|$ over the range $-5 \leq \frac{x}{L} \leq 5$, but when 
reconstructing the object field we use samples that span the range $-20 \leq \frac{x}{L} \leq 20$ ]. Choosing of $T / L=0.3745$ results in a sampling rate $f_{c}=530,\left[f_{c} L=1.335\right.$ in Fig. 1], thus we have ensured that $f_{c}<f_{x}$. This sampling process produces an array [size $N=1068$ ] of complex values which denote $u_{z}^{s}(n)$. Performing a numerical Fourier transform on $u_{z}^{s}(n)$ produces another array $U_{z}^{s}(n)$ [size $N=1068$ ], whose magnitude we also plot in Fig. 1. By comparing $\left|U_{z}^{s}(n)\right|$ and the actual analytical signal $|U(v)|$, it is clear that significant aliasing has occurred and the two distributions are markedly different from each other.

Using a trapezoidal integration formula with the array $u_{z}^{s}(n)$ we numerically calculate the inverse Fresnel transform to give $u^{s}(X)$. In Fig. 3(b) we plot $\left|u^{s}(X)\right|$ over the range $-5 \leq X / L \leq 5$. We can see the object field is accurately reconstructed, specifically we have recovered the spatial frequency, $f_{x}$ even though the field $u_{z}(x)$ was sampled at a rate $f_{c}$ such that $f_{c}<f_{x}$. We also note that each replica, SR, of $u^{s}(X)$ is separated by an amount $(\lambda z / T) / L$, in keeping with the prediction made by Eq. (6).

\section{PRACTICAL LIMITATIONS}

In this section we examine how practical implementation issues may limit the application of the generalized sampling theory in DH systems, particularly for fields that contain spatial frequencies higher than the sampling rate of the camera. In Section 3.1 we discuss the effect the presence the twin image has on the reconstructed object field. It is shown that unless the twin image is removed, the object field cannot be reconstructed without significant aliasing. In Section 3.2 we assume that the twin image can be separated from the desired real image using PSI and so it would appear that once again that the theory outlined in Section 2 can be employed. This is subject to certain qualifications: the generalized sampling theory presented in Section 2 assumes that the diffracted field is sampled at discrete points. However, in practical systems the intensity of the field incident upon the camera is averaged over the pixel area. We examine the implications of this averaging operation for practical DH systems and conclude that the highest spatial frequency recoverable may ultimately be limited by noise.

\subsection{Presence of the twin image}

Following, ${ }^{36}$ in Section 2 it was assumed that the complex amplitude of the real image could be recorded at the camera plane. In fact recovering the complex amplitude typically involves several more steps which can be implemented either during the experiment or afterwards using numerical processing. ${ }^{5,9}$ For the inline DH setup we are considering a known reference field, $u_{r}$, interferes with the propagated object field, $u_{z}(x)$, at the camera plane encoding the phase information as intensity variations. The total complex field at the camera plane, $u_{c}(x)$ is thus written as

$$
u_{c}(x)=u_{r}+u_{z}(x)
$$

where we define

$$
u_{r}=A \exp (j \alpha)
$$

and explicitly write

$$
u_{z}=\left|u_{z}(x)\right| \exp [j \phi(x)] .
$$

With Eq. (15) and Eq. (16), the intensity recorded by the camera is given by

$$
\begin{aligned}
& I_{c}=\left|u_{c}\right|^{2}=u_{c} \times u_{c}^{*} \\
& I_{c}=|A|+\left|u_{z}(x)\right|^{2}+u_{r} u_{z}^{*}(x)+u_{r}^{*} u_{z}(x)
\end{aligned}
$$

where $*$ indicates the complex conjugate operator.

The first two terms in Eq. (17) are the dc terms, while we refer to the third and fourth terms as the real and twin image respectively. The plane wave reference field can be removed by dc filtering the Fourier transform (performed numerically in a computer) of the captured image using numerical techniques. It is good practice 

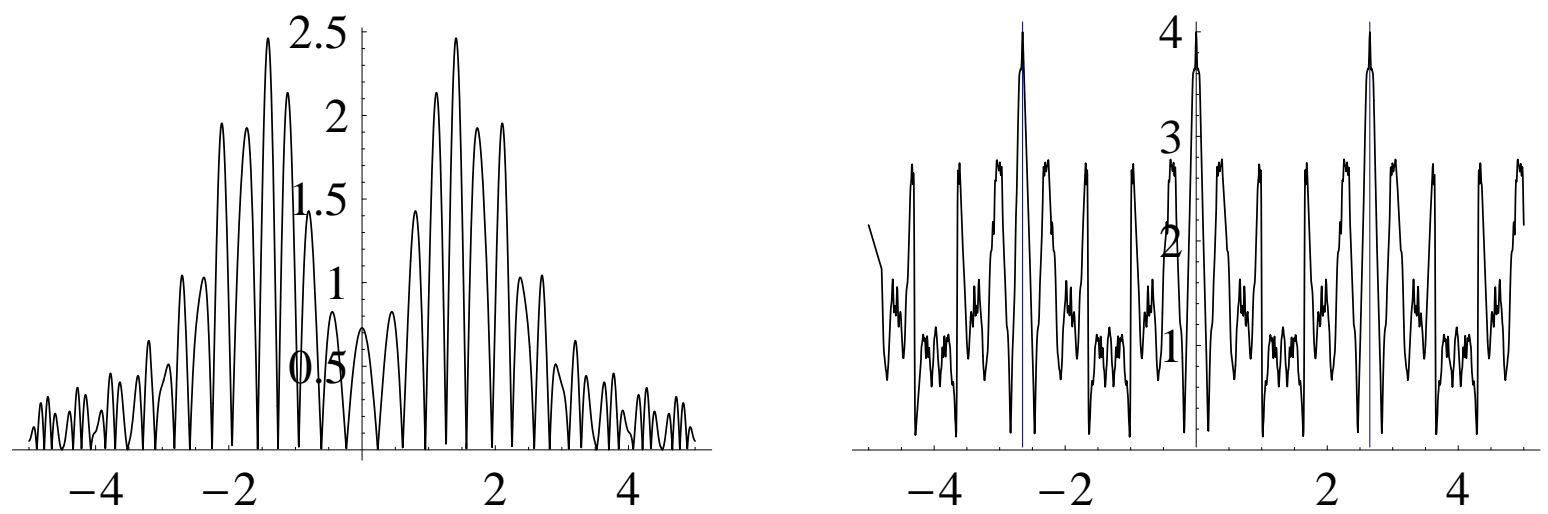

Figure 4. Same as Figure 3 but with twin image included.

to ensure that $\left|u_{r}\right|>>\left|u_{z}(x)\right|$ and so $\left|u_{z}(x)\right|^{2} \approx 0$ when compared to the magnitude of the real and twin images. To ease analysis we will make a series of simplifications: (i) First we note that $u_{z}^{*}(x)=u_{-z}(-x)$, (ii) Let $\left|u_{r}\right|>>\left|u_{z}(x)\right|$ and so we neglect $\left|u_{z}(x)\right|^{2}$, (iii) We assume the dc term $|A|^{2}$ has been filtered out numerically and finally (iv) We neglect the constant phase terms $\exp (j \alpha)$ and $\exp (-j \alpha)$ that precede $u_{z}^{*}(x)$ and $u_{z}(x)$ respectively in Eq. (17), to give

$$
I_{c} \approx A u_{-z}(-x)+A u_{z}(x) .
$$

Using the same simulation values as in Section 2.2 we present the equivalent plot, this time however with the twin image included. It can be seen by comparing Fig. 3(a) and Fig. 4(a) that the image recorded by the camera in both situations is different. This is even more apparent when we attempt to reconstruct the object field from the captured hologram, the result of which can be seen in Fig. 4(b). Clearly aliasing occurs which significantly reduces the quality and detail of the reconstructed image. It may be possible to reduce the magnitude of the twin image using numerical techniques ${ }^{37}$ which is currently being investigated.

\subsection{Averaging effect of finite size pixels}

As we have just noted in Section 3.1 the presence of the twin image has a significant impact for the practical implementation of the theory presented in Section 2. In order to be able to gain access to the potential sampling advantages afforded by the general sampling theory it is thus necessary to remove the twin image. One possible method for removing the twin image is PSI. ${ }^{23,29}$ In this section we first review how PSI can be used to recover the real complex valued image and then we examine what role pixel averaging has on the recovery of high spatial frequencies.

PSI techniques generally rely on capturing several intensity images, where the reference field is phase stepped by a known amount between each capture. Using a numerical algorithm an estimate of the wrapped phase can be calculated. ${ }^{9}$ For simplicity let us assume that our reference field is again given by Eq. (15). Let us further assume that the form of the propagated object field incident upon our camera is given by $u_{p w}(x)=\exp \left[j \phi_{p w}(x)\right]$, where $\phi_{p w}(x)=2 \pi f_{x} x$. Using a standard 4-step numerical algorithm the wrapped phase, $\phi_{w}(x)$ may then be estimated using the formula ${ }^{9,38}$

$$
\phi_{w}(x)=\arctan \left\{\frac{-\left[I_{\alpha=\frac{3 \pi}{2}}(x)-I_{\alpha=\frac{\pi}{2}}(x)\right]}{I_{\alpha=0}(x)-I_{\alpha=\pi}(x)}\right\},
$$


where $I_{\alpha}$, for this particular example, is given

$$
\begin{aligned}
I_{\alpha}(x) & =\left(|A|^{2}+1\right)+I_{\alpha}^{a c}, \\
I_{\alpha}^{a c}(x) & =2|A| \cos \left[\phi_{p w}(x)-\alpha\right] .
\end{aligned}
$$

Let us now consider how the camera records the intensity incident upon it. A camera consists of an array of $N \times N$ pixels separated from each other by a distance $T$ - we assume a $100 \%$ fill factor. We can represent (in $1-\mathrm{D})$ the intensity array using the vector

$$
I_{\alpha}^{n}=\left[I_{\alpha}^{1}, I_{\alpha}^{2}, \ldots \ldots . . I_{\alpha}^{N}\right] .
$$

Suppose we wish to find the intensity at some point $x=x_{1}$ where the point $x_{1}$ is located exactly in the center of pixel $n$. The camera will represent the intensity $I_{\alpha}\left(x_{1}\right)$ as $I_{\alpha}^{n}$ where

$$
\begin{aligned}
I_{\alpha}^{n} & =\int_{x_{1}-T / 2}^{x_{1}+T / 2} I_{\alpha}(x) d x \\
I_{\alpha}^{n} & =\left(|A|^{2}+1\right)+2|A| \cos \left[\phi_{p w}\left(x_{1}\right)-\alpha\right] \times \frac{\sin \left[\phi_{p w}\left(x_{1}\right)-\alpha\right]}{\phi_{p w}\left(x_{1}\right)-\alpha}
\end{aligned}
$$

which simplifies to

$$
I_{\alpha}^{n}=\left(|A|^{2}+1\right)+I_{\alpha}^{a c}\left(x_{1}\right) \operatorname{sinc}\left[2 \pi\left(\frac{T}{2}\right) f_{x}-\alpha\right],
$$

where

$$
\operatorname{sinc}(x)=\frac{\sin (x)}{x} .
$$

The results derived in Eq.'s (23) to (25) imply: (i) The intensity value recorded by the camera is weighted by its spatial frequency, (ii) at spatial frequencies that are large relative to the pixel size, noise will become increasingly important in determining the accuracy of the measurement. For example with a pixel size of $7.4 \mu \mathrm{m}$ the first null of the weighting sinc function is reached at a spatial frequency of $136,000 \mathrm{~m}^{-1}$. A typical camera with $1000 \times$ 1000 pixels, corresponding active area $7.4 \mathrm{~mm} \times 7.4 \mathrm{~mm}$ has a bandwidth of $135,135 \mathrm{~m}^{-1}$. From this analysis it would appear that as the spatial frequency of the signal increases, the camera's ability to record it decreases. The ability of a camera to discern a spatial frequency from random noise fluctuations will limit ultimately the maximum recoverable spatial frequency.

\section{CONCLUSION}

In this manuscript we have reviewed some of the more recent developments in sampling theory and their implications for practical Digital Holographic (DH) systems. We began in Section 2 by re-deriving a general sampling rule that has been presented in the literature by several different authors. ${ }^{28,30,31}$ We then explored some of the implications of this theory by comparing the numeric and analytical representations of a special case analytical solution. It was shown that although the numerical Fourier transform $\left(\left|U_{z}^{s}\right|\right)$ of the sampled the Fresnel distribution, $u_{z}(x)$ was significantly aliased and differed markedly from its analytical representation it could nevertheless be used to reconstruct the object field correctly. In Section 3 we discussed some practical issues that arise when implementing inline DH systems. It was shown that unless the twin image can be removed significant aliasing will occur in the reconstructed object wave-field. It may be possible to use other numerical techniques to remove the twin image and this is currently being investigated further by the group. A well-known technique for separating the dc terms and the twin image from the real image - Phase Shifting Interferometry (PSI) - was also examined. Currently it is assumed that the field sampled by the camera at discrete points only, however the finite size of the pixels and the intensity averaging that occurs over their area ensures that this assumption is an idealization. We examined some of the implications of sampling the diffracted field above the Nyquist limit and found that systemic noise in the system may ultimately limit the application of the general sampling theory in practical digital holographic systems. 


\section{ACKNOWLEDGMENTS}

We would like to thank Lukas Ahrenberg for fruitful discussions. The research leading to these results has received funding from the European Community's Seventh Framework Programme FP7/2007-2013 under grant agreement no. 216105.

\section{REFERENCES}

1. D. Gabor, "A new microscope principle," Nature 161, pp. 777-778, 1948.

2. E. N. Leith and J. Upatnieks, "Reconstructed wavefronts and communication theory," J. Opt. Soc. Am. 52, p. 1123, 1962.

3. E. N. Leith and J. Upatnieks, "Wavefront reconstruction with continuous-tone objects," J. Opt. Soc. Am. 53, p. 1377, 1963.

4. O. Bryngdahl and A. W. Lohmann, "Interferograms are image holograms," J. Opt. Soc. Am. 58, pp. 141$142,1968$.

5. P. Hariharan, Basics of holography, Cambridge, 2002.

6. J. W. Goodman and R. Lawrence, "Digital image formation from electronically detected holograms," Appl. Phys. Lett. 11, pp. 77-79, 1967.

7. L. P. Yaroslavskii and N. S. Merzlyakov, Methods of Digital Holography, Consultants Bureau, 1980.

8. T. M. Kreis, M. Adams, and W. P. O. Juptner, "Methods of digital holography: a comparison," Proc. SPIE 3098, pp. 224-233, 1997.

9. U. Schnars and W. Juptner, "Digital recording and numerical reconstruction of holograms," Meas. Sci. Technol. 13, pp. 85-101, 2002.

10. D. P. Kelly, B. Hennelly, W. Rhodes, and J. T. Sheridan, "Analytical and numerical analysis of linear optical systems," Opt. Eng. 45, p. 008201, 2006.

11. B. M. Hennelly and J. T. Sheridan, "Generalizing, optimizing, and inventing numerical algorithms for the fractional fourier, fresnel, and linear canonical transforms," J. Opt. Soc. Am. A 22, pp. 917-927, 2005.

12. B. M. Hennelly and J. T. Sheridan, "Fast algorithm for the linear canonical transform," J. Opt. Soc. Am. A 22, pp. 928-937, 2005.

13. J. Goodman, Introduction to Fourier Optics, 2nd ed., McGraw-Hill, New York, 1966.

14. J. Cooley and J. Tukey, "An algorithm for the machine calculation of complex fourier series," Math. Comput. 19, pp. 297-301, 1965.

15. R. Bracewell, The Fourier Transform and its Applications, McGraw-Hill, New York, 1965.

16. Y. Takaki and H. Ohzu, "Fast numerical reconstruction technique for high-resolution hybrid holographic microscopy," Appl. Opt. 38, pp. 2204-2211, 1999.

17. G. Pedrini, P. Frning, H. Tiziani, and F. Santoyo, "Shape measurement of microscopic structures using digital holograms," Appl. Opt. 164, pp. 257-268, 1999.

18. S. Schedin, G. Pedrini, H. Tiziani, A. Aggarwal, and M. Gusev, "Highly sensitive pulsed digital holography for built-in defect analysis with a laser excitation," Appl. Opt. 40, pp. 100-117, 2001.

19. T. Kreis, M. Adams, and W. Juptner, "Digital in-line holography in particle measurement," Proc. SPIE 3744, pp. 54-64, 1999.

20. S. Murata and N. Yasuda, "Potential of digital holography in particle measurement," Opt. and Laser Tech. 32, pp. 567-574, 2000.

21. T. Kreis and W. Juptner, "Suppression of the dc term in digital holography," Opt. Eng. 36, pp. 2357-2360, 1997.

22. Y. Surrel, "Design of algorithms for phase measurements by the use of phase stepping," Appl. Opt. 35, pp. 51-60, 1996.

23. I. Yamaguchi and T. Zhang, "Phase-shifting digital holography," Opt. Lett. 22, pp. 1268-1270, 1997.

24. H. Nyquist, "Certain topics in telegraph transmission theory," Trans. AIEE 47, pp. 617-644, 1928.

25. C. E. Shannon, "Communication in the presence of noise," Proc. Institute of Radio Engineers 37, pp. 10-21, 1949. 
26. E. Whittaker, "On the functions which are represented by the expansions of the interpolation theory," Proc. Royal Soc. Edinburgh, Sec. A, 35, pp. 181-194, 1915.

27. V. Kotelnikov, "On the carrying capacity of the ether and wire in telecommunications," Material for the First All-Union Conference on Questions of Communication, Izd. Red. Upr. Svyazi RKKA, Moscow, 1933.

28. F. Gori, "Fresnel transform and sampling theorem," Opt. Eng. 39, pp. 293-297, 1981.

29. A. Stern and B. Javidi, "Sampling in the light of wigner distribution: errata," J. Opt. Soc. Am. A 21, pp. 2038-2038, 2004.

30. A. Stern and B. Javidi, "Analysis of practical sampling and reconstruction from fresnel fields," Opt. Eng. 43, pp. 239-250, 2004.

31. L. Onural, "Sampling of the diffraction field," Appl. Opt. 39, pp. 5929-5935, 2000.

32. A. Stern, "Sampling of linear canonical transformed signals," Sig. Proc. 86, pp. 1421-1425, 2006.

33. C. Candan and H. Ozaktas, "Sampling and series expansion theorems for fractional fourier and other transforms," Sig. Proc. 83, pp. 2455-2457, 2003.

34. R. T. B. Li and Y. Wang, "New sampling formulae related to linear canonical transform," Sig. Proc. 86, pp. 983-990, 2007.

35. J. J. Healy and J. T. Sheridan, "Cases where the linear canonical transform of a signal has compact support or is band-limited," Opt. Lett. 33, pp. 228-230, 2008.

36. A. Stern and B. Javidi, "Improved-resolution digital holography using the generalized sampling theorem for locally band-limited fields," J. Opt. Soc. Am. A 23, pp. 1227-1235, 2006.

37. T. Latychevskaia and H.-W. Fink, "Solution to the twin image problem in holography," ArXiv Physics e-prints, Oct. 2006.

38. I. Yamaguchi and T. Zhang, "Phase-shifting digital holography," Opt. Lett. 22(16), pp. 1268-1270, 1997. 